\title{
An event detection framework for the representation of the AGGIR variables
}

\author{
José Manuel Negrete Ramírez ${ }^{*}$, Philippe Roose ${ }^{\dagger}$, Marc Dalmau $^{\ddagger}$ \\ IUT de Bayonne - LIUPPA \\ Université de Pau et des Pays de l'Adour \\ Anglet, France \\ *jose-manuel.negrete-ramirez@ univ-pau.fr \\ $\dagger\{$ Philippe.Roose $\} ;{ }^{\ddagger}\{$ Marc.Dalmau $\} @$ iutbayonne.univ-pau.fr
}

\author{
Yudith Cardinale \\ Dpto. de Computación y \\ Tecnología de la Información \\ U. Simón Bolívar \\ Venezuela \\ ycardinale@usb.ve
}

\begin{abstract}
In this paper, we propose a framework to study the AGGIR (Autonomy Gerontology Iso-Resources Groups) grid model, in order to evaluate the level of independency of elderly people, according to their capabilities of performing activities and interact with their environments over the time. To model the Activities of Daily Living (ADL), we also extend a previously proposed Domain Specific Language (DSL), in order to employ operators to deal with constraints related to time and location of activities, and event recognition. Our framework aims at providing an analysis tool regarding the performance of elderly/handicapped people within a home environment by means of data recovered from sensors using the iCASA simulator. To evaluate our approach, we pick three of the AGGIR variables (i.e., dressing, toileting, and transfers) and evaluate their testability in many scenarios, by means of records representing the occurrence of activities of the elderly. Results demonstrate the accuracy of our framework to manage the obtained records correctly and thus generate the appropriate event information.
\end{abstract}

Index Terms-Domain Specific Language, Feature-oriented Programming, Pervasive Computing, Pervasive Health Systems and Services, AGGIR grid

\section{INTRODUCTION}

According the United Nations Department of Economic and Social Affairs (UN DESA) ${ }^{1}$, population is aging faster than ever before. Due to this increase, reducing medical costs and improving quality of care service [1] have become in recent years a need for new care delivery mechanisms and structures [2]. Personal Sensor Networks (PSN) and Body Sensor Networks (BSN) in smart environments have become a viable alternative to traditional healthcare solutions. PSNs are used to detect human daily activities and measure conditions within the environment. BSNs are used to monitor vital signs and health conditions by measuring physiological parameters.

Several approaches propose different frameworks focusing on identifying the Activities of Daily Living (ADL's) that require monitoring [3], [4], [5], [6], [7], [8], [9]. However, there is still a lack of ADL activities to be analysed, whereas others consider a subset of specific activities; but most importantly, they are not based on a specific tool such as the AGGIR (Autonomy Gerontology Iso-Resources Groups [10] grid. The AGGIR grid [10] is an autonomy assessment tool used in France to measure the indenpendncy level of elderly

\footnotetext{
${ }^{1}$ https://www.un.org/development/desa/publications/world-populationprospects-the-2017-revision.html
}

people. It is a six-level dependence scale (GIR1 to GIR6), that can be defined based on a set of seventeen three-state variables. Each variable can have one of these values: $\mathbf{A}$, for complete dependency; $\mathbf{B}$, for partial dependency; and $\mathbf{C}$, for complete independency. The variables are classified into two groups: discriminatory and illustrative variables. Table I provides an overview for those variables previously mentioned.

TABLE I: AGGIR variables

\begin{tabular}{|c|c|}
\hline $\begin{array}{c}\text { Discriminatory } \\
\text { Variables }\end{array}$ & Illustrative variables \\
\hline Coherence & Management \\
\hline Location & Cooking \\
\hline Toileting & Housekeeping \\
\hline Dressing & Transportation \\
\hline $\begin{array}{l}\text { Self-feeding / } \\
\text { Alimentation }\end{array}$ & Purchases \\
\hline Elimination & Medical treatment \\
\hline Transfers & Leisure activities \\
\hline $\begin{array}{c}\text { Indoor } \\
\text { movement }\end{array}$ & \\
\hline $\begin{array}{l}\text { Outdoor } \\
\text { movement }\end{array}$ & \\
\hline $\begin{array}{c}\text { Distant } \\
\text { communication }\end{array}$ & \\
\hline
\end{tabular}

In this context, in a previous work [11] we have proposed a general framework for the architecture of a context aware service delivery. Afterworth, in [12] a DSL which allows the representation of the activities composing the AGGIR grid in the form of a plot, providing a history file for detecting abnormal behavior of the inhabitants. In this work, we extend our previous works, by proposing a framework that integrates an exteded DSL to achieve the monitoring of a person within a smart home environment to identify the activities performed by the inhabitants over a certain period of time, by means of collection and analysis of data obtained from sensors located in the environment. In order to address such a task, we extend our previous proposed DSL language by defining operators, which deal with constraints related to time, location, and event recognition. Moreover, this DSL will be able to categorise a set of activities based on the constants of the AGGIR grid [10], which classifies autonomy levels to various environmental factors affecting the activities and social life of a person.

Our framework aims at providing an analysis tool regarding the performance of elderly/handicapped people within a home environment by means of data recovered from sensors using the iCASA simulator. To evaluate our approach, we pick three 
of the AGGIR variables (i.e., dressing, toileting, and transfers) and evaluate their testability in many scenarios, by means of records representing the occurrence of activities or unexpected behavior of the elderly. Results demonstrate the accuracy of our framework to manage the obtained records correctly and thus generate the appropriate event information.

The remainder of this paper is organized as follows. Related studies are presented in Section II. A brief review of operators regarding time, location, and event constraints is described in Section III. Our proposal is explained in Section IV. The details of the experiments and the discussion about obtained results are given in Section V. Finally, conclusions and future works are given in Section VI.

\section{STATE OF THE ART}

In this section, we survey studies focused on detecting activities in smart homes for elderly people.

In [3], it is proposed the Context-Aware Service Integration System (CASIS), a framework aimed to improve the quality of care of elderly people by providing context-aware services and healthcare services by means of a variety of home appliances and sensor technology. The framework uses Web services and OSGi [13] gateways to provide external support and support at home, respectevely. The work studies how to improve the quality of care by providing context-aware services and healthcare services by means of smartphones and a reminder system. The system provides a variety of services for the elder, as well as related services for family, friends, healthcare professionals, and external service providers. Connecting all caring services together in a service-oriented framework, CASIS coordinates them to provide personalized services. CASIS provides context-dependent services employing a rule-based reasoning approach and an inference agent. The OSGI home gateway manages the environment and serves as a portal for the outside connection. The targeted activities proposed by [3] are limited to location, mealtime, and taking medications, and the AGGIR model is not considered. It is focused on the welfare of the elderly subject's lifestyle by means of a smart telephone and a reminder system. In contrast, our framework is able to detect activities related to the AGGIR model.

An approach, based on ambient-intelligence compliant objects (AICOs), was studied by [4]. AICOs is able to classify human activities of daily living and to identify how many activities can be detected simultaneously using a BayesianNetwork-based fusion engine. It integrates event-driven services with the OSGi service platform. In order to collect data, sensors were integrated into home objects used by the inhabitant; succeding in the identification of an activity map of human living and including a floor object to identify the subject's location, as well a power object to measure the usage of electronic devices. Nevertheless, the list of sensors in the living environment is only applicable to a single subject, not considering the ADL's that affect the abilities of the subject and dependency level. In our proposed framework we consider ADL's as described by the AGGIR variables.

The work proposed in [5] (Smart Auto-Dep) provides a framework to evaluate a subjects dependency. The proposed service-oriented architecture is based on the activities of daily living defined by the AGGIR scale [10]. It proposes a contextaware monitoring and evaluation for assessing the dependency level of the elderly; in order to guarantee an appropriate level of monitoring and services. The authors work with a set of heterogeneous sensors and equipment such as GPS, single inertial sensors, light switches, door fridge, door sensors, and IP cameras, for monitoring ADL. The objective is to express all the activities considered in the AGGIR model such as moving, eliminating, eating, dressing. They integrated raw sensor data, by means of a $\mathrm{Cilia}^{2}$ model built upon OSGi and several communication interfaces like Bluetooth, WiFi, Zigbee, and proprietary norms.

A home monitoring system (Wellness) is presented in [6] to monitor and evaluate the well-being of elderly subjects performing ADL's alone. The system relies on a time-series analysis of household appliance usage, connected through various sensors attached to various appliances and connected to a wireless sensor network using a ZigBee module. ADL's like sleeping, meal preparation, eating, going to the toilet, grooming, and watching TV are identified based on the frequency and duration time. Additionally, the wellness functions are checked, based on the activity of specific appliances. However, the system does not provide adequate or in-depth knowledge about the elderly inhabitant or how, emergency alerts could be raised if necessary.

The work in [7] proposes a system to estimate activities of daily living using a method based on 3D acceleration sensors and ZigBee networks. The system is composed of two accelerometer sensor tags which are attached to the subject's body (hand and waist) and send data to the server via an access point or a coordinator. The Received Signal Strength Indication (RSSI) of the sensor is used to calculate the position of the device by detecting the distance between the sensor devices and the access point. Motion is estimated based on acceleration data. It uses fuzzy inference and area recognition (based on RSSI) and selects the fuzzy membership function and the rules according the result of area recognition. Eleven motions are selected from ADL's for evaluation. The complexity of estimating the activities is highlighted; however the approach is limited to three ADL's in the living area. In our framework, the activities can be detected all over the house.

The work of [8] proposes an ADL detection system using small, low-cost, non-intrusive, wrist-worn sensors embedded in a normal sports watch: an accelerometer, a temperature, and an altimeter sensor. The watch communicates with the computer via a USB RF access point. The targeted activities include walking, sleeping, washing dishes, and watching TV. The accuracy was achieved by means of a SVM (Support Vector Machines) classification algorithm for detecting the activities of the elderly subject. Some system confusions were detected among various activities, such as ironing, brushing teeth, and washing dishes. In fact, the system supposes that those activities have some characteristics in common, which leads to confusion of the classification process.

The Context Aware Real-time Assistant (CARA) [9] is

\footnotetext{
${ }^{2}$ http://wikiadele.imag.fr/index.php/Cilia
} 
a context-aware hybrid reasoning framework that integrates fuzzy rule-based reasoning with Case-Based Reasoning (CBR) for pervasive healthcare in smart home environments. It processes information uncertainty and uses context to provide context-aware sensor data fusion as well as anomaly detection mechanisms that supports ADL analysis and alert generation. In CARA, the raw data is transformed into low-level context data and built case queries and fuzzy sets. Afterwards, the case-based and fuzzy rule-based reasoning work simultaneously. The case-based cycle performs anomaly detection and home automation by means of four operations: retrieval, reuse, revision, and retention. The fuzzy rule-based cycle identifies the current situation as normal, abnormal, or emergency after generating a high-level abstract context data. Regardless of the proposed hybrid context-aware reasoning system, the efficiency evaluation was confined to a limited set of activities.

A more comprehensive study is presented in [14], where detailed information and classification of Health Monitoring Systems (HMS) and healthcare applications are provided. HMS in smart environments are reviewed from a general perspective and with a particular focus on systems for the elderly and dependent people.

All these works focused on identify correctly the activities that require monitoring. However, there is still a lack of ADL activities to be analysed, whereas others consider a subset of specific activities; but most importantly, they are not based on a specific tool such as the AGGIR grid. We overcome these limitations by offering a framework based on a DSL for identifying the activities of the elderly inhabitant based on the parameters of the AGGIR system, which is currently the legal tool in France for measuring the autonomy of the elderly.

\section{TEMPORAL, LOCALIZATION, AND EVEnTS OPERATORS: A REVIEW}

In this section, we present a review of different operators used to describe time and location relations among events. Actions and activities that can be identified in a space are called events. These events can be tagged with time and location, that in turns relate them.

According to Cook et al. [15], in order for a system to make sensible decisions, it has to be aware of where the users are and have been during some period of time. Spatial and Temporal logic is a well established area of Artifitial Intelligence (AI) [16], which has been applied to represent and reason on spatial and temporal features and constraints of context and situations [17]. For instance, temporal knowledge on human activities can be specified by means of the temporal operators ANDlater and ANDsim in Event-Condition-Action rules introduced by Augusto et al. [18]. Gottfried et al. [19] apply Allen's Temporal Logic [20] (Table II) to describe, constrain, and reason on temporal sequences in dealing temporal and spatial knowledge in smart homes as well.

The event calculus of Shanahan [21] infers what is true from information that expresses what and when something occurs (actions) and what happens after those actions. Shanahan uses the action concept instead of event, but it makes no difference between the two. The notion of fluent is used to express anything that can change over time, as seen on Table III.
TABLE II: Allen's temporal operators

\begin{tabular}{|c|c|c|c|}
\hline Relation & Symbol & Symbol for inverse & Illustration \\
\hline $\mathrm{X}$ before $\mathrm{Y}$ & $i$ & $i$ (after) & $\underline{X}-\quad Y$ \\
\hline $\mathrm{X}$ equals $\mathrm{Y}$ & $=$ & $=$ (equals) & - \\
\hline $\mathrm{X}$ meets $\mathrm{Y}$ & $\mathrm{m}$ & mi (met by) & $\underline{\underline{X}} \quad \underline{Y}$ \\
\hline X overlaps Y & o & oi (overlapped by) & $-\underline{X}-\underline{Y}$ \\
\hline $\mathrm{X}$ during $\mathrm{Y}$ & $\mathrm{s}$ & di (contains) & $\frac{X}{Y}$ \\
\hline $\mathrm{X}$ starts $\mathrm{Y}$ & $\mathrm{d}$ & si (started by) & $-\underline{X}_{\underline{Y}}$ \\
\hline $\mathrm{X}$ finishes $\mathrm{Y}$ & $\mathrm{f}$ & fi (finished by) & $\underline{Y} \underline{X}$ \\
\hline
\end{tabular}

TABLE III: Shanahan's event calculus formulas

\begin{tabular}{|c|l|}
\hline Formula & \multicolumn{1}{c|}{ Meaning } \\
\hline Initiates $(\alpha, \beta, \tau)$ & Fluent $\beta$ starts to hold after action $\alpha$ at time $\tau$ \\
\hline Terminates $(\alpha, \beta, \tau)$ & Fluent $\beta$ ceases to hold after action $\alpha$ at time $\tau$ \\
\hline InitiallyP $(\beta):$ & Fluent $\beta$ holds from time 0 \\
\hline$\tau_{1} \leq \tau_{2}$ & Time point $\tau_{1}$ is before time point $\tau_{2}$ \\
\hline Happens $(\alpha, \tau)$ & Action $\alpha$ occurs at time $\tau$ \\
\hline HoldsAt $(\beta, \tau)$ & Fluent $\beta$ holds at time $\tau$ \\
\hline $\operatorname{Clipped}\left(\tau_{1}, \beta, \tau_{2}\right)$ & Fluent $\beta$ is terminated between times $\tau_{1}$ and $\tau_{2}$ \\
\hline
\end{tabular}

Some others works worth revising are shown on Table IV, where operators concerning temporal, spatial, and eventrelated operators are proposed in order to provide a significant contribution to a solution of these problems. We consider the temporal operators proposed by Allen [20], as well as operators such as "Before, During, After, Begin, End, startTime, endTime". With regard to location operators, we employ "Inside, Outside, Joint, nextTo", among others. We also utilise conventional mathematic operators such as "greater than, lower than", to indicate event conditions based on attributes.

TABLE IV: Spatial, temporal and event operators

\begin{tabular}{|l|c|c|c|}
\cline { 2 - 4 } \multicolumn{1}{c|}{} & Temporal & Location & Event \\
\hline Freksa [22] & $\nabla$ & $\square$ & $\square$ \\
\hline Ribarić and Dalbelo Bašić [23] & $\square$ & $\square$ & $\square$ \\
\hline Randell et al. [24] & $\square$ & $\square$ & $\square$ \\
\hline Galton [25] & $\square$ & $\square$ & $\square$ \\
\hline Randell et al. [26] & $\square$ & $\square$ & $\square$ \\
\hline Kim et al. [27] & $\square$ & $\square$ & $\square$ \\
\hline Pecora et al. [28] & $\square$ & $\square$ & $\square$ \\
\hline Bruno et al. [29] & $\square$ & $\square$ & $\square$ \\
\hline Patkos et al. [30] & $\square$ & $\square$ & $\square$ \\
\hline Santos et al. [31] & $\square$ & $\square$ & $\square$ \\
\hline Furze and Bennett [32] & $\square$ & $\square$ & $\square$ \\
\hline Angsuchotmetee et al. [33] & $\square$ & $\square$ \\
\hline
\end{tabular}

\section{A FRAMEWORK TO EVALUATE THE AGGIR CONSTANTS: OUR APPROACH}

In this section, we describe our framework and the extension of the DSL. Fig. 1 shows the architecture of our framework, composed by three components: (i) the Descriptor module, (ii) the Simulator module, and (iii) the Analyzer module. All of them suported by the DSL.

\section{A. Framework modules}

The Descriptor module provides a Graphic User Interface (GUI) where all the criteria over the time dimension, location, and events are described as well as the required scenarios for 


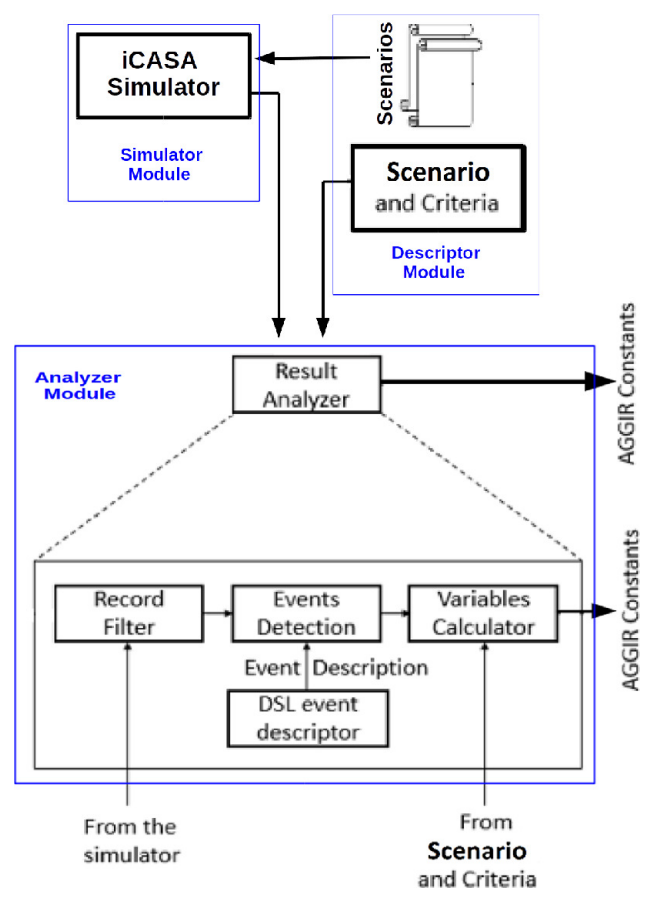

Fig. 1: General Architecture of the proposed framework

the simulation. Also, sensors to identify the ADLs have to be specified through the GUI. We propose a group of sensors that can describe such activities. Those ones are enlisted in Table $\mathrm{V}$, where some examples of their use are proposed.

In the Simulator module, the activities performed by the subject are carried out and information is recovered from sensors located within the smart home environment. As the Simulator module, we have integrated iCASA [34] to our framework, which is a smart home simulator which allows to have control over: time, environment, inhabitants, devices, a graphical user interface, scripting facilities (for the environment), and notification facilities [35]. iCASA is used in order to set up a simulated scenario. The purpose of employing iCASA, which is based on OSGi [13] and iPOJO [36], is that it takes advantage of the versatility and dynamism of the two latter, which are provided as a set of modules and components (e.g., bundles and iPOJO components) that are deployed on a OSGi/iPOJO framework.

TABLE V: Sensors for activity recognition

\begin{tabular}{|c|}
\hline $\begin{array}{c}\text { Home sensors } \\
\text { devices }\end{array}$ \\
\hline Presence sensor \\
\hline Thermometer \\
\hline Heater \\
\hline Light \\
\hline Door-Window \\
\hline Flood Sensor \\
\hline Tablet \\
\hline Motion sensor \\
\hline Siren \\
\hline Push button \\
\hline Camera \\
\hline Airflow (Breathing) Sensor \\
\hline Blood Pressure Sensor \\
\hline CO / CO 2 sensor \\
\hline Cooler \\
\hline Sprinkler \\
\hline Audio source \\
\hline
\end{tabular}

The Analyzer module analyzes all the collected data in order to classify them and evaluate if the AGGIR variables of the case study have been carried out to completion. The Analyzer consists of: (i) a Record filter, which organizes the resulting data into a set of records; (ii) an Event detector, for the detection of the performed activities; and (iii) a Variable calculator, for determining whether the AGGIR variables have been performed successfully or not. In order to obtain information to achieve the identification of the AGGIR constants after every activity has been carried out to completion, some relevant data have to be considered, as shown in Table VI. All these data are specified in the Descriptor module. After the simulation, a history log file is created, which considers the logical aspect of situations, i.e., if the inhabitant is eating and its noon, it might seem logical, but the fact that the inhabitant is eating in the toilet is not logical.

TABLE VI: Additional data for each activity

\begin{tabular}{|c|c|}
\hline Extra data type for each task & Data type \\
\hline startTime & Date / String \\
\hline endTime & Date / String \\
\hline Duration & Integer/Double \\
\hline Location & String \\
\hline Day & String / Integer \\
\hline
\end{tabular}

As a result, the history log file is necessary to deduct the possible activities which will be performed in the future, and to find a relationship among them to assure a coherent behaviour. Such a file will permit to obtain information whether it is from long or short periods of time; making possible the identification of complex situations such as displacement inside the home environment, where data collected through the timeline of activities is useful to determine if the behaviour of the inhabitant can be considered as normal.

\section{B. Main characteristics of the proposed DSL}

The proposed DSL allows to express situations related to the AGGIR constants that respond to the activities performed by people at home with a physical or mental disability, support for elderly, diseases connected to aging. With the DSL is also possible to describe the data that one wishes to recover from the various sensors presented within a smart home environment and the operations related to them.

The DSL permits the representation of attribute-based event conditions. Through the GUI of the Descriptor module, the description of targeted events from an operation perspective, can be done. Such an operation consists in measuring one of the following options relying on event conditions based on atributes (greater, equal, less, etc.): a value, a maximum or minimum value, an average of a set of values. The operations described below are employed for developing the aforementioned interface in the DSL: (i) The Value operation: which returns the value (s) of the device; (ii) The Maximum operation: which returns the maximum value of the value(s) of the device; (iii) The Minimum operation: returns the minimum of the value (s) of the device; (iv) The Average operation: which returns the average of the value (s) of the device.

Spatio-temporal event conditions are also represented by the DSL. Those are defined by temporal operators such as "Before, During, After, Begin, End", and those defined using spatial operators i.e., "Inside, Outside, Joint". The proposed criteria to determine the parameters for the example of the precedent 
paragraph, are: time and space, and within the time dimension, we can also find (i) Concurrency: for recognizing activities which take place simultaneously, but they do not necessarily require the users interaction at the same time. That is, activities that have been started but not yet ended by the inhabitant [37]; (ii) Precedence: for establishing a logical order of the activities, i.e., going to the bathroom, and then washing his hands; (iii) Simultaneity: for identifying which activity takes most of the time from the user, when multitasking capabilities might be present, i.e., preparing meal and calling on the phone; or watching television while eating. (iv) Recurrence: for determining a logical sequences of situations. In the case where there is a recurrence of an activity, it is essential to define at what this activity is carried out regularly. Some examples are presented on Table VII.

TABLE VII: Criteria proposed over the time dimension

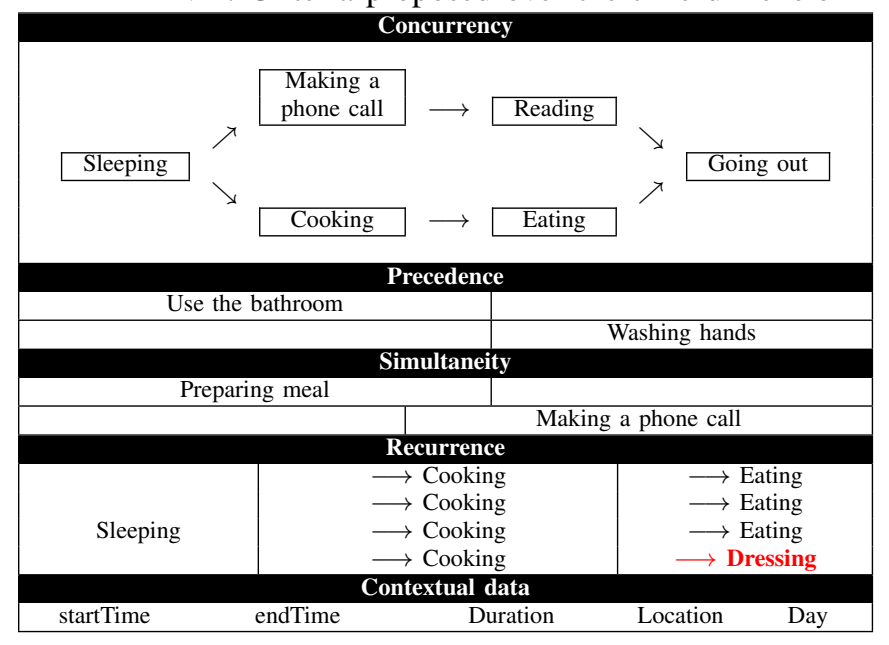

V. Use CASE DESCRIPTION

To evaluate our framework, we propose a set of experiments, where three AGGIR variables are concerned: Dressing, Toileting, and Transfers. The dressing variable assesses the ability to wear clothing, upper and lower body parts of the subject. The toileting variable monitors the ability to maintain personal hygiene. The transfers variable measures the possibility of getting up, lying down, and sitting, as part of daily activities. The difficulty in determining the values of these variables lays on the fact that some can be measured directly, such as sitting or lying down, whereas some others, such as toileting, have a complex context to determine, based on the values of a number of sensors within a limited time range.

The importance of the extension of the DSL relays on its potential to describe events that can be indirectly observed within the time domain. The "indirect" term in this context means that there is no sensor to measure value inmediately, but it rather refers to collecting and analyzing the readings of a number of sensors to calculate a value associated with a complex event. For example, toileting is associated with a number of simple activities that are performed within a limited time range, such as toilet use and hand washing. There is not a standard approach to measuring such activities; but it can be estimated on average according to the existing routines of the inhabitant.

\section{A. Simulation steps}

The simulation steps for the proposed framework are described in the next paragraphs:

Step 1. The first step is to specify the criteria over the time and location of activities, type and location of sensors, and events that have to be detected (in this case, all activities related to Dressing, Hygiene, and Transfers), and prepare the scenario for an elderly indoor daily routine over the course of one week. All this is done in GUI of the Descriptor module. The primary source of information used to generate the scenario is the schedule proposed by the work of [9] but many modifications take place in order to make the scenario more suitable for the simulation. However, the XML format is used to describe the scenario programmatically. By means of XML, it is possible to describe events chronologically so the Simulator module can process these files.

Step 2. Then, the Simulator module, i.e., the iCASA framework, executes the simulation according to the information provided by the Descriptor module.

Step 3. After the simulation is performed, the record filter component of the Analyzer module organizes the resulting data into a set of records, each of which represents an action captured by a sensor within the smart home environment (see Fig. 1). Each record consists of data fields, such as the time it occurred (according to the simulator clock), the sensor ID and the sensed value. These are raw data and need to be examined by the Analyzer module in order to generate the detection of events and then calculate the values of the AGGIR variables. The next step is the detection of events. An event is a composite act that is observed using more than one sensor, which means that an event has more than one record in raw data. The DSL is used to provide an accurate and unified description of the events. Once the event detection has ocurred, the variables calculator within the Analizer module indicates whether an AGGIR variable has been accomplished or not.

To illustrate this, we offer as example of an event related to toileting: when the toilet is used, the inhabitant should wash his hands after such an action is finished. In order to analyze this event, the records created by the presence of the sensor in the bathroom, along with those ones issued by the use of the toilet flush and the washbasin, must be examined. See Table VIII. for the description of all activities related to the three considered AGGIR variables and sensors related to each one. Because of the nature of the AGGIR variables, there is an urgent need to set up a criteria to be applied to generate the analysis of such events. If such criteria is met, then the so-called variable will take a positive value that means the elderly has the ability to perform the activity/event that is being evaluated; otherwise, it means that the individual does not have the ability to carry out such a task.

Although every variable in the AGGIR grid is based on three major states, which identify that the elderly can either possess the ability to perform the activities concerning one variable, whether it is completely, partially, or not existent at all; this study covers a proposition where only two cases out of three AGGIR variables are accomplished by the inhbitant, meaning that whether they are in complete possession of the skills for 
TABLE VIII: Orchestration of activities / AGGIR Constants

\begin{tabular}{|c|c|c|}
\hline \multicolumn{3}{|c|}{$\begin{array}{l}\text { Definitions } \\
\text { Constant = Situation }\end{array}$} \\
\hline Toileting & Dressing & Transfers \\
\hline \multicolumn{3}{|c|}{ Situation $=\sum(+)$ Activities } \\
\hline Open bathroom door & Open wardrobe door & Lying down \\
\hline Use of toilet flush & $\begin{array}{l}\text { Spend time changing } \\
\text { clothes }\end{array}$ & Sitting down \\
\hline $\begin{array}{c}\text { Wash hands } \\
\text { Close bathroom door }\end{array}$ & Close wardrobe door & Getting up \\
\hline \multicolumn{3}{|c|}{ Activity $=\sum(+)$ Sensor data } \\
\hline $\begin{array}{l}\text { Bathroom door sensor } \\
\text { (On-Off / Boolean) }\end{array}$ & $\begin{array}{l}\text { Wardrobe door sensor } \\
\text { (On-Off / Boolean) }\end{array}$ & $\begin{array}{l}\text { Bed capacitive } \\
\text { sensor (On-Off / } \\
\text { Boolean) }\end{array}$ \\
\hline $\begin{array}{c}\text { Toilet flush sensor } \\
\text { (On-Off / Boolean) }\end{array}$ & $\begin{array}{c}\text { Wardrobe proximity } \\
\text { sensor }(\text { On-Off / } \\
\text { Double) }\end{array}$ & $\begin{array}{c}\text { Chair capacitive } \\
\text { sensor (On-Off / } \\
\text { Boolean) }\end{array}$ \\
\hline $\begin{array}{c}\text { Washbasin proximity } \\
\text { sensor }(\text { On-Off / Double) }\end{array}$ & & \\
\hline
\end{tabular}

perfoming the activities composing the evaluated variable or simply not. We are currently working on including the three states as future work.

To calculate the toileting variable the following conditions must be met: the person must have a bath four times a week at least, wash his hands after eating or using the toilet, for at least three times a day.

To calculate the transfer variable, namely the ability of a person to perform the basic movements of his dayly routine, such as rising from bed, sitting down and standing up from a chair; we consider that there must be at least three sitting events a day, either taking place in the living room or in the kitchen, and at least rising from the bed one time per day.

To verify if the dressing variable is achieved, dressing events like approching the wardrobe must be encountered at least twice a day.

\section{B. Experimental Results}

All simulating and processing operations were performed by means of two sets of inputs for our framework, in order to generate two different scenarios. we separate the week into seven days, each of which is represented using one file within the smart home environment.

The first scenario is a simulation of an ideal week where all the activities were performed by the inhabitant with no impediment into seven files, one file for each day. The activities of the elderly were monitored using a simulated sensor network in the house environment. In Table IX, we show per day the number of activities detected, related to each considered AGGIR variable. The check mark means that the criterio to consider that the person is independent (i.e., minimum number of activities related to each variable) has been reached.

TABLE IX: Results of the first scenario.

\begin{tabular}{|c|c|c|c|c|}
\hline & & Transfer & Toileting & Dressing \\
\hline \multirow{8}{*}{ 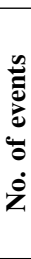 } & MON & ( 7 & V 8 & $\sqrt{6} 3$ \\
\hline & TUE & $\nabla 6$ & $\nabla 8$ & V 3 \\
\hline & WED & 『 7 & $\nabla 11$ & $\nabla 2$ \\
\hline & THU & घ 7 & $\nabla 10$ & $\nabla 2$ \\
\hline & FRI & v 8 & च 8 & V 3 \\
\hline & SAT & $\square 8$ & $\nabla 6$ & $\nabla 4$ \\
\hline & SUN & $\nabla 6$ & $\nabla 9$ & $\nabla 3$ \\
\hline & Total & $\nabla 49$ & $\nabla 59$ & $\begin{array}{ll} & 19\end{array}$ \\
\hline
\end{tabular}

In the second scenario, we deliberately dropped some daily activities in a way that we can cause a malfunctioning on the criteria we developed, the days were randomly chosen, as shown on Table X.

TABLE X: Results of the second scenario.

\begin{tabular}{|c|c|c|c|c|}
\hline & Transfer & Toileting & Dressing \\
\hline \multirow{8}{*}{ 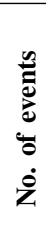 } & MON & $\square 7$ & ه 7 & $\nabla 3$ \\
\hline & TUE & $\checkmark 7$ & 『 7 & $\otimes 0$ \\
\hline & WED & $\checkmark 7$ & $\nabla 11$ & $\nabla 2$ \\
\hline & THU & $\nabla 6$ & $\nabla 11$ & $\nabla 3$ \\
\hline & FRI & $\square 8$ & $\nabla 8$ & $\nabla 3$ \\
\hline & SAT & 凶3 & $\nabla 6$ & $\nabla 4$ \\
\hline & SUN & $\nabla 6$ & $\nabla 6$ & $\nabla 2$ \\
\hline & Total & $\nabla 44$ & $\nabla 56$ & $\nabla 16$ \\
\hline
\end{tabular}

The detection method within the event detector and the variable calculator does not rely on the number of simple events when calculating the variables, but it depends on the generation of complex events.

The experiment was designed to examine three concepts: the first is calculating the constants of the AGGIR grid: dressing, transfer, and toileting. using the suggested method. The second is the scalability, where we expand the time frame to verify the ability of the framework to deal with larger time domains and thousands of records and events. Finally, we examined algorithm performance for different simulation times.

Firstly, the method succeeded in identifying three simulated problems within a week. The days were chosen randomly. Fig. 2 and Fig. 3 show that despite the number of records in the second scenario related to personal transfer and toileting did not change significantly compared to the first analyzed scenario, however a problem with the dressing variable was detected. This, in turn, reflects the how the DSL event descriptor can perform a smart analysis of events.

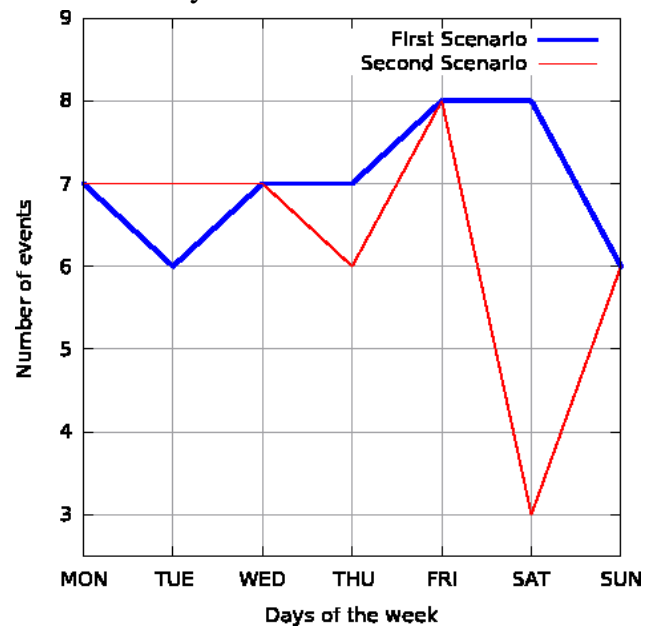

Fig. 2: Transfer events

Subsequently, we tested the scalability of the framework in a time domain for a period of time longer than one week. For this matter, we simulated three months and generated the corresponding records. Next, we applied the criteria used to create the events, and finally, we measured the values of the previous three constants. We assumed that each month consisted of thirty days, which allowed flexibility when processing the records and generating events. By doing so, there is a possibility to use the actual number of days within the 


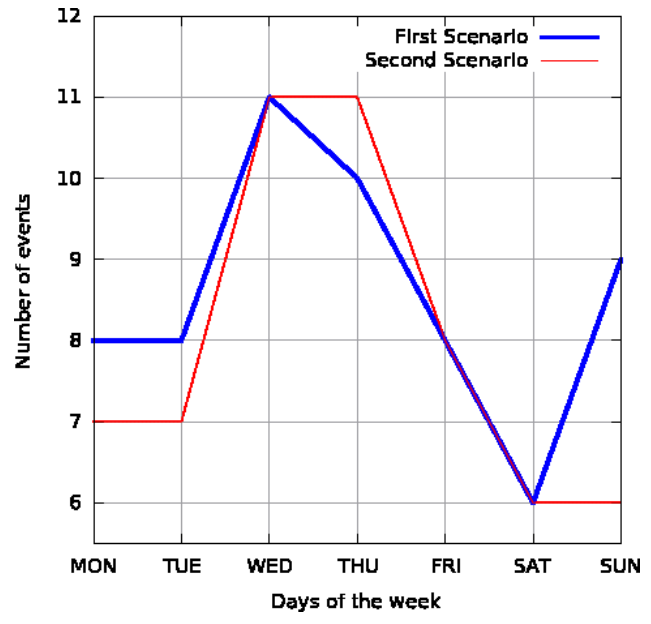

Fig. 3: Toileting events months, i.e., 28, 29, 30 and 31 days (Fig. 4), requiring further processing. Finally, to make sure that the algorithm is scalable, we monitored the time elapsed to calculate the constants in order to simulate a day, a week, two weeks, three weeks, a month, two months, and three months. Table XI shows the elapsed time for each case. The values displayed include the time spent for each simulation, as well as results analysis, events generation, and finally the calculation of the constants variable.

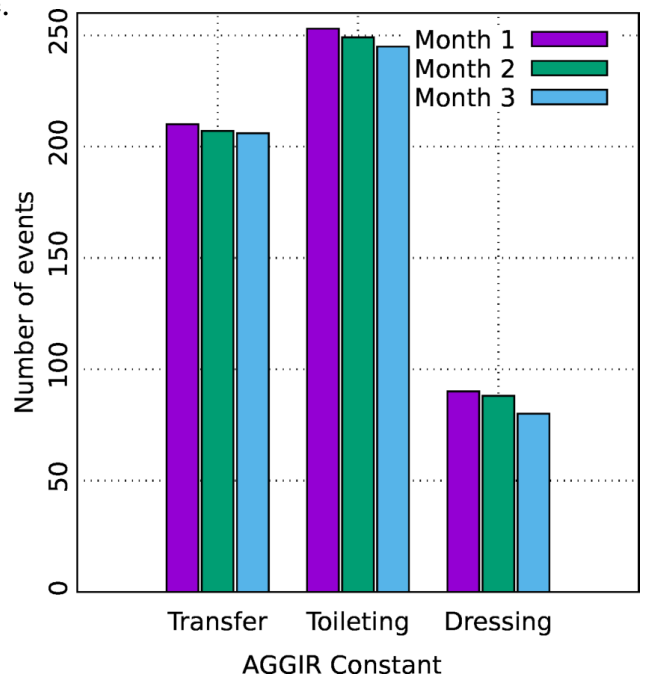

Fig. 4: Three-month algorithm scalability

TABLE XI: Real time elapsed to calculate the constants in seven different simulation time ranges (Day, $\boldsymbol{W}$ eek, $\boldsymbol{M}$ onth).

\begin{tabular}{|c|c|c|c|c|c|c|c|}
\hline Time period & $1 \boldsymbol{D}$ & $1 \boldsymbol{W}$ & $2 \boldsymbol{W}$ & $3 \boldsymbol{W}$ & $1 \boldsymbol{M}$ & $2 \boldsymbol{M}$ & $3 \boldsymbol{M}$ \\
\hline No. of days & 1 & 7 & 14 & 21 & 30 & 60 & 90 \\
\hline Time $(\boldsymbol{s})$ & 62 & 434 & 867 & 1301 & 1863 & 3713 & 5590 \\
\hline
\end{tabular}

Fig. 5 shows the performance of the algorithm with simulating scenarios with different time ranges. The resulting diagram is linear, which corresponds to the complexity of the algorithm of the Analyzer module, represented by $O(n)$, where $n$ represents the number of events, meaning that the framework as a whole, is scalable.

\section{CONCLUSION}

In this paper, we present a framework based on an extension of a previous proposed Domain Specific Language (DSL),

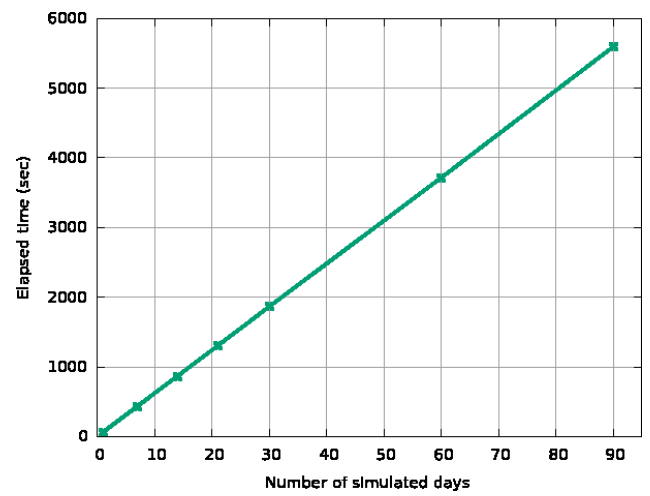

Fig. 5: Analysis of Algorithm performance

which uses operators in order to deal with time and location of activities and event detection constraints. Our framework is intended to describe activities performed by a subject within a smart home environment and to identify the AGGIR variables. We based our approach on The AGGIR grid, a tool for determining the autonomy of an inhabitant adopted by the French government, The results of two simulated scenarios are analyzed using the previous methodology in order to examine the criteria we developed. Additionally, we tested the scalability of the framework, to make a three-month evaluation. Future work includes the extension of the approach to study other AGGIR variables and to consider their three states, and the development of other case studies in order to adjust the approach and validate it further.

\section{REFERENCES}

[1] U. Varshney, "Pervasive healthcare," Computer, vol. 36, no. 12 , pp. 138-140, 2003.

[2] M. N. Kamel Boulos, R. C. Lou, A. Anastasiou, C. D. Nugent, J. Alexandersson, G. Zimmermann, U. Cortes, and R. Casas, "Connectivity for healthcare and well-being management: examples from six european projects," Int. J. of environmental research and public health, vol. 6, no. 7, pp. 1947-1971, 2009.

[3] W.-r. Jih, J. Y.-j. Hsu, C.-L. Wu, C.-F. Liao, and S.-y. Cheng, "A multi-agent service framework for contextaware elder care," in In AAMAS, 2006.

[4] C.-H. Lu and L.-C. Fu, "Robust location-aware activity recognition using wireless sensor network in an attentive home," IEEE Trans. on Automation Science and Engineering, vol. 6, no. 4, pp. 598-609, 2009.

[5] T. Lemlouma, S. Laborie, and P. Roose, "Toward a context-aware and automatic evaluation of elderly dependency in smart homes and cities," in Int. Symp. on World of Wireless, Mobile and Multimedia Networks. IEEE, 2013, pp. 1-6.

[6] N. K. Suryadevara and S. C. Mukhopadhyay, "Wireless sensor network based home monitoring system for wellness determination of elderly," IEEE Sensor Journal, vol. 12, no. 6, pp. 1965-1972, 2012.

[7] E. Shimokawara, T. Kaneko, T. Yamaguchi, M. Mizukawa, and N. Matsuhira, "Estimation of basic 
activities of daily living using zigbee $3 \mathrm{~d}$ accelerometer sensor network," in ICBAKE. IEEE, 2013, pp. 251-256.

[8] S. Chernbumroong, S. Cang, A. Atkins, and H. Yu, "Elderly activities recognition and classification for applications in assisted living," Expert Systems with Applications, vol. 40, no. 5, pp. 1662-1674, 2013.

[9] B. Yuan and J. Herbert, "Context-aware hybrid reasoning framework for pervasive healthcare," Personal and ubiquitous computing, vol. 18, no. 4, pp. 865-881, 2014.

[10] E. Dupourqué, S. Schoonveld, and J. B. Bushey, "Aggir, the work of grids," Long-term Care News, vol. 32, 2012.

[11] J. M. N. Ramírez, P. Roose, and M. Dalmau, "Distributed interfaces and context-oriented broadcast services in a smart-home environment," in Int. Conf. on Wireless and Mobile Computing, Networking and Communications. IEEE, 2016, pp. 1-8.

[12] J. M. N. Ramírez, P. Roose, M. Dalmau, and M. Bakni, "Proposal and validation of a domaine specific language for the representation of the aggir constants." in HEALTHINF, 2018, pp. 438-445.

[13] O. Alliance, "Osgi alliance: Osgi service platform, core specification, release 4, version 4.2," OSGi Alliance, 2009.

[14] H. Mshali, T. Lemlouma, M. Moloney, and D. Magoni, "A survey on health monitoring systems for health smart homes," Int. J. of Industrial Ergonomics, vol. 66, pp. 2656, 2018.

[15] D. J. Cook, J. C. Augusto, and V. R. Jakkula, "Ambient intelligence: Technologies, applications, and opportunities," Pervasive and Mobile Computing, vol. 5, no. 4, pp. 277-298, 2009.

[16] L. Liao, D. Fox, and H. Kautz, "Extracting places and activities from gps traces using hierarchical conditional random fields," Int. J. of Robotics Research, vol. 26, no. 1, pp. 119-134, 2007.

[17] J. Ye, S. Dobson, and S. McKeever, "Situation identification techniques in pervasive computing: A review," Pervasive and mobile computing, vol. 8, no. 1, pp. 3666, 2012.

[18] J. C. Augusto, J. Liu, P. McCullagh, H. Wang, and J.-B. Yang, "Management of uncertainty and spatio-temporal aspects for monitoring and diagnosis in a smart home," Int. J. of Computational Intelligence Systems, vol. 1, no. 4, pp. 361-378, 2008.

[19] B. Gottfried, H. W. Guesgen, and S. Hübner, "Spatiotemporal reasoning for smart homes," in Designing Smart Homes. Springer, 2006, pp. 16-34.

[20] J. F. Allen, "Maintaining knowledge about temporal intervals," in Readings in qualitative reasoning about physical systems. Elsevier, 1990, pp. 361-372.

[21] M. Shanahan, "The event calculus explained," in Artificial intelligence today. Springer, 1999, pp. 409-430.

[22] C. Freksa, "Temporal reasoning based on semi-intervals," Artificial intelligence, vol. 54, no. 1-2, pp. 199-227, 1992.

[23] S. Ribarić and B. Dalbelo Bašić, "Modelling crisp and fuzzy qualitative temporal relations," Journal of Infor- mation and Organizational Sciences, vol. 25, no. 2, pp. 81-91, 2001.

[24] D. A. Randell, Z. Cui, and A. G. Cohn, "A spatial logic based on regions and connection.” 1992.

[25] A. Galton, "Lines of sight," in AISB Workshop on Spatial and Spatio-Temporal Reasoning, 1994.

[26] D. Randell, M. Witkowski, and M. Shanahan, "From images to bodies: Modelling and exploiting spatial occlusion and motion parallax," in IJCAI, 2001, pp. 57-66.

[27] J. Kim, H.-s. Choi, H. Wang, N. Agoulmine, M. J. Deerv, and J. W.-K. Hong, "Postech's u-health smart home for elderly monitoring and support," in Int. Symp on World of Wireless Mobile and Multimedia Networks. IEEE, 2010, pp. 1-6.

[28] F. Pecora, M. Cirillo, F. Dell'Osa, J. Ullberg, and A. Saffiotti, "A constraint-based approach for proactive, context-aware human support," J. of Ambient Intelligence and Smart Environments, vol. 4, no. 4, pp. 347-367, 2012.

[29] B. Bruno, J. Grosinger, F. Mastrogiovanni, F. Pecora, A. Saffiotti, S. Sathyakeerthy, and A. Sgorbissa, "Multimodal sensing for human activity recognition," in Int. Symp. on Robot and Human Interactive Communication. IEEE, 2015, pp. 594-600.

[30] T. Patkos, D. Plexousakis, A. Chibani, and Y. Amirat, "An event calculus production rule system for reasoning in dynamic and uncertain domains," Theory and Practice of Logic Programming, vol. 16, no. 3, pp. 325-352, 2016.

[31] P. E. Santos, M. F. Martins, V. Fenelon, F. G. Cozman, and H. M. Dee, "Probabilistic self-localisation on a qualitative map based on occlusions," J. of Experimental \& Theoretical Artificial Intelligence, vol. 28, no. 5, pp. 781-799, 2016.

[32] T. A. Furze and B. Bennett, "Using the principles of classical conditioning to learn event sequences," Computational Models Of Cognitive Development, pp. 40-47, 2011.

[33] C. Angsuchotmetee, R. Chbeir, Y. Cardinale, and S. Yokoyama, "A dynamic event detection framework for multimedia sensor networks," in Asia-Pacific Conference on Communications. IEEE, 2017, pp. 1-6.

[34] P. Lalanda, C. Hamon, C. Escoffier, and T. Leveque, "icasa, a development and simulation environment for pervasive home applications," in Consumer Communications and Networking Conference. IEEE, 2014, pp. 1142-1143.

[35] P. Lalanda, J. A. McCann, and A. Diaconescu, Autonomic computing. Springer, 2013.

[36] C. Escoffier, R. S. Hall, and P. Lalanda, "ipojo: An extensible service-oriented component framework," in Int. Conf. on Services Computing. IEEE, 2007, pp. 474 481.

[37] R. Helaoui, M. Niepert, and H. Stuckenschmidt, "Recognizing interleaved and concurrent activities: A statisticalrelational approach," in $n t$. Conf. on Pervasive Computing and Communication. IEEE, 2011, pp. 1-9. 